\title{
Male mate choice and insemination success under simultaneous versus sequential choice conditions
}

\author{
Megan L. Head*, Frances Jacomb, Regina Vega-Trejo, Michael D. Jennions \\ Division of Evolution, Ecology and Genetics, Research School of Biology, Australian National University, Acton, Canberra, Australia
}

\section{A R T I C L E I N F O}

\section{Article history:}

Received 11 December 2014

Initial acceptance 19 January 2015

Final acceptance 5 February 2015

Available online 13 March 2015

MS. number: 14-01008

\section{Keywords:}

dichotomous choice

mate encounter rate

mating success

no-choice

Poeciliid

sequential choice

sexual selection

simultaneous choice

sperm allocation
Theory predicts that males should be choosier when encountering potential mates simultaneously rather than sequentially because there is no opportunity cost. Consequently, when mate encounter rates vary across space and time males might benefit from plasticity in mate preferences to match prevailing social conditions, preferring high-quality mates when females are encountered frequently and showing no preferences when females are encountered rarely. Here we investigated how encounter type (i.e. simultaneous or sequential) alters male mate preferences for female size in the mosquitofish, Gambusia holbrooki. We found that male mosquitofish attempted to mate with a relatively large female significantly more often than a relatively small female when presented with two females simultaneously. In contrast, males showed no such preference when sequentially presented with two females. Further, males attempted more copulations with absolutely larger females irrespective of encounter type. Despite these behavioural patterns, however, neither male insemination success nor the number of sperm transferred was influenced by female size or the encounter type. Our results provide support for the prediction that male mate choice is stronger during simultaneous choice encounters, but suggest that insemination success in G. holbrooki is partly under female control.

(c) 2015 The Association for the Study of Animal Behaviour. Published by Elsevier Ltd. All rights reserved.
Male mate choice is more likely to evolve when there is variation in female quality, males have limited resources to invest in mating and there are low costs to being choosy (Bonduriansky, 2001; Edward \& Chapman, 2011). To date, most empirical studies of male mate choice have focused on identifying the targets of choice (e.g. Pack et al., 2009; Tigreros, Mowery, \& Lewis, 2014) and the benefits associated with choosing particular females (e.g. LeBas, Hockham, \& Ritchie, 2003; Kekalainen, Huuskonen, Tuomaala, \& Kortet, 2010; Nordeide, Kekäläinen, Janhunen, \& Kortet, 2013). These studies have highlighted that male mate choice can evolve in a broad range of mating systems. There is, however, far less understanding of what contributes to variation in the presence and the strength of male mate choice among populations and between species (but see Dougherty \& Shuker, 2014).

A key factor in the evolution of male mate choice is the relationship between the number of receptive females (i.e. mate encounter rate) and a male's capacity to mate. Male mate choice is

\footnotetext{
* Correspondence: M. L. Head, Division of Evolution, Ecology and Genetics, Research School of Biology, Australian National University, Acton, Canberra, 0200 Australia.

E-mail address: megan.1.head@gmail.com (M. L. Head).
}

predicted to evolve when mate availability is high and male capacity to mate repeatedly is low (Edward \& Chapman, 2011). If females are frequently encountered, it is even possible that two or more potential mates are encountered simultaneously. This makes male mate choice more likely as individuals can choose between the immediately available mates at no cost (i.e. rejection does not lower the mating rate). Consequently, even small differences in the profitability of each mating favour the evolution of choice. In contrast, during sequential encounters, choosiness lowers a male's mating rate because some females are rejected (Barry \& Kokko, 2010). Simultaneous availability of mates is a general cue that mate encounter rates are likely to be high.

It is expected that when mate availability/encounter rates vary across space and time individuals should adjust their level of choosiness to the perceived mate availability (Svensson, Lehtonen, \& Wong, 2010). Under low mate availability, such that mates are only sequentially encountered, individuals should take advantage of a current mating opportunity. Under high mate availability, especially if this leads to simultaneous encounters with mates, individuals should be choosier. This prediction is best studied in experiments that compare male choice in different social contexts to control for effects of variation in male 'time out' on choosiness. For example, experimental studies on fiddler crabs (Uca spp.) have 
shown that males do not discriminate between heterospecifics and conspecifics during sequential encounters but do during simultaneous encounters (Booksmythe, Jennions, \& Backwell, 2011). Likewise, male sticklebacks, Gasterosteus aculeatus, and male salamanders, Desmognathus santeetlah, preferred to court larger females, but only when females were presented simultaneously rather than sequentially (Rowland, 1982; Verrell, 1995). This trend is widespread. Interestingly, however, when examining all available studies greater choosiness during simultaneous choice is observed for females, but not for males, indicating that males across species may respond less consistently than females to variation in encounter rate (meta-analysis: Dougherty \& Shuker, 2014). Male mate choice involves decisions not only about whether to mate, but also how to allocate resources to each mating (Parker, 1998; Parker \& Pizzari, 2010). For example, males can vary how much sperm they transfer depending on a female's size, condition or mating history (meta-analysis: Kelly \& Jennions, 2011). Consequently it can be informative to look not only at mating behaviour but also at insemination success and the number of sperm transferred to different females. How social environments influence male allocation of sperm to females of different quality has mostly been studied in the context of sperm competition (review: Wedell, Gage, \& Parker, 2002). Theory predicts that males should adjust sperm allocation in response to the risk and intensity of competition (review: Parker \& Pizzari, 2010; meta-analysis: Kelly \& Jennions, 2011). Less is known about how males adjust sperm allocation to other social cues. More specifically, there are few studies designed to directly compare sperm allocation under different mate encounter scenarios (for a noteworthy exception see Cornwallis \& Birkhead, 2006). However, greater sperm allocation to highquality females has been shown for males exposed to females both simultaneously (e.g. two-choice tests: Cornwallis \& Birkhead, 2006) and sequentially (e.g. 'no-choice' tests: Lüpold, Manier, AlaHonkola, Belote, \& Pitnick, 2010; Rubolini et al., 2006; see also Appendix S2 of: Kelly \& Jennions, 2011). These studies suggest that males can allocate sperm strategically, even during sequential mate choice.

Here we investigated how encounter type (i.e. simultaneous or sequential) affects male mate preferences for larger females in the mosquitofish, Gambusia holbrooki. Mosquitofish are well suited to investigating the causes of variation in male mate choice. First, they have internal fertilization and males transfer sperm to females via a modified anal fin called the gonopodium (Constanz, 1989). Males do not engage in courtship but perform coercive 'sneak' copulations in which they approach a female from behind and thrust their gonopodium towards her gonopore (Bisazza, 1993; Bisazza \& Marin, 1995). This occurs repeatedly, which makes it possible to quantify male mating attempts (e.g. Booksmythe, Backwell, \& Jennions, 2013). Second, female size varies considerably and is strongly correlated with fecundity (Bisazza, Marconato, \& Marin, 1989; Callander, Backwell, \& Jennions, 2012; Deaton, 2008). Thus, there are clear benefits to mating with larger females. Despite the likely benefits, however, male preferences for large females are not universal: some studies show a male preference for larger females (Bisazza et al., 1989; Callander et al., 2012; Mautz \& Jennions, 2011), and others do not (McPeek, 1992). Furthermore, studies show that male preferences for large females can vary depending on other factors (e.g. trial type: Hoysak \& Godin, 2007; mating history: VegaTrejo, O'Dea, Jennions \& Head, 2014). Third, males invest considerable effort trying to mate (attempting to copulate up to 20 times/ min; Wilson, 2005) and may often make mate choice decisions when sperm stores are low (O'Dea, Jennions, \& Head, 2014). Consequently, pursuing low-quality females could be costly in terms of lost opportunities to inseminate more profitable females. Finally, mosquitofish have highly dynamic social groups, forming mixed-sex shoals of varying size and sex ratio (Agrillo, Dadda, \& Serena, 2008). The social environment varies widely, with the adult sex ratio and density of each sex changing throughout the breeding season (e.g. Kahn, Kokko, \& Jennions, 2013). As such, males experience considerable variation in female encounter rates. Selection for plastic changes in mating behaviour given different mate encounter rates might therefore be strong.

Owing to the potential for individuals to encounter prospective mates simultaneously, studies of male mate choice in mosquitofish have only employed designs that use 'two-choice' (simultaneous) trials, measuring male association time with females presented behind dividers (e.g. Mautz \& Jennions, 2011; Wong \& McCarthy, 2009) and/or recording attempted sneak copulation rate in trials in which males can interact freely with two females (e.g. Hoysak \& Godin, 2007; Vega-Trejo et al., 2014). To our knowledge there have been no experiments using 'no-choice' (i.e. sequential) mating trials to investigate male mate choice in $G$. holbrooki. It is therefore unknown whether males adjust their mate choice based on female encounter rate. These rate changes are exemplified at the extremes by simultaneous versus sequential encounters with receptive females.

In our experiment we independently manipulated mate encounter type and the relative size of the focal females encountered. We investigated how these two factors influenced male mate choice behaviour (number of attempted copulations) towards a focal female, insemination success (whether or not the female is inseminated) and sperm allocation (how many sperm are transferred). We predicted that (1) males will show a preference for relatively larger females and (2) if the mate encounter rate strongly influences the costs of choice then male preferences will be stronger during simultaneous than sequential trials. If the effects are weak, however, males should show a similar preference for relatively large females regardless of encounter type.

\section{METHODS}

\section{Origin and Maintenance of Fish}

Male fish were collected from two ponds $\left(35^{\circ} 14^{\prime} 27^{\prime \prime} \mathrm{S}\right.$, $149^{\circ} 5^{\prime} 27^{\prime \prime} \mathrm{E}$ and $35^{\circ} 14^{\prime} 13^{\prime \prime} \mathrm{S}, 149^{\circ} 5^{\prime} 55^{\prime \prime} \mathrm{E}$ ) in Canberra, Australia, in February 2014 . The females used were first-generation laboratoryreared fish whose parents were collected from the same ponds in March 2013. Prior to the experiment all fish were housed in singlesex tanks at densities of 30-60 fish per 90 litres, and females were thus virgins. Fish were maintained at $27^{\circ} \mathrm{C}$ on a $14: 10 \mathrm{~h}$ light:dark cycle and fed Artemia salina nauplii and commercial fish flakes twice daily. Males were kept in the laboratory for 3-6 months prior to being used in our experiment.

\section{Experimental Design}

Each male was exposed to two females, one of which was the focal female. We independently manipulated (1) how males encountered the focal female (sequentially or simultaneously with the other female) and (2) the relative size of focal females (bigger or smaller than the other female). We then investigated the effects of these two factors and their interaction on male mate choice and sperm allocation using a $2 \times 2$ factorial design. Thus, we had four experimental treatments (sequential/relatively small female, sequential/relatively large female, simultaneous/relatively small female, simultaneous/relatively large female). Each male was assigned a unique pair of females and was only used once.

To manipulate the relative size of focal females we divided virgin females from our stock population into three size classes: small (<300 mg), medium (350-450 mg) and large (>500 mg). Female 
weight was highly correlated with standard length measured from photographs taken after trials $(r=0.938, P<0.001, N=288)$. In our experimental trials the medium-sized female was always the focal female. Males in the relatively small focal female treatment were paired with a medium and a large female, whereas males in the relatively large focal female treatment were paired with a medium and a small female. Our manipulation of the focal female's relative rather than absolute size ensured that any difference in how males responded to a focal female was not confounded by her absolute size.

We also manipulated how females were presented to males to investigate whether male mate choice decisions depend on whether they encounter mates simultaneously or sequentially. In our simultaneous treatment both the focal female and the nonfocal female were placed in experimental tanks with the male on day 0 and remained with him for the duration of the experiment. In our sequential treatment only the nonfocal female was placed with the male on day 0 . On day 6 immediately prior to behavioural observations we replaced the nonfocal female with the focal female.

\section{Experimental Protocol}

A schematic of the experimental protocol is given in Fig. 1. Prior to each experimental trial both sexes were anaesthetized in ice slurry. Excess water was removed from females which were then placed in a dish of water on a Mettler Toledo balance to measure wet weight (to the nearest $\mathrm{mg}$ ). Males were stripped of sperm (details below) so that they all began the experiment with fully depleted sperm reserves.

Once fish had recovered, males were placed in one-half of a 7litre aquarium $(17 \times 28 \mathrm{~cm}$ and $15 \mathrm{~cm}$ deep $)$ that was divided in half by a mesh barrier. At the same time, depending on the treatment, the appropriate combination of females was placed in the other half of the tank. Focal females for the sequential choice treatment were kept separately in individual 1-litre tanks until needed for behavioural trials. On day 3 males were briefly taken out of their treatment tank and their ejaculates were again stripped. This allowed us to check whether male sperm number varied with the treatment a male experienced while replenishing sperm reserves. Previous studies have shown that the social conditions in which males are housed can influence sperm production (i.e. sperm priming: Aspbury \& Gabor, 2004; Barrett, Evans, \& Gasparini, 2014). If male sperm reserves differed between our treatments this could influence interpretation of our subsequent results. However, as there was no effect of treatment on sperm number at day 3 (quasi-Poisson generalized linear model, GLM: encounter type: $t_{1,112}=0.446, P=0.657$; relative size of focal female: $t_{1,112}=0.302, P=0.763$; interaction: $\left.t_{1,112}=-1.605, P=0.111\right)$, we do not consider this further.

\section{Behavioural Observations}

After males had spent a further 3 days in their respective treatments (total of 6 days), we conducted behavioural observations of male mate choice. For our sequential choice treatment the nonfocal female was replaced with the focal female immediately before the behavioural observations. To begin an observation session we removed the mesh barrier and allowed males and females to interact freely. We allowed fish to acclimate for 2 min before recording their behaviour. We recorded the number of mating attempts towards the focal female (see Vega-Trejo et al., 2014). Behavioural observations lasted for $10 \mathrm{~min}$ and the fish were then allowed to interact for another $20 \mathrm{~min}$. After $30 \mathrm{~min}$ we extracted sperm from the focal female's reproductive tract.

\section{Collecting Sperm from Males}

To ensure that male sperm number did not differ depending on our treatment (see above) sperm were stripped from males following the methods of Matthews, Evans, and Magurran (1997). Briefly, males were placed on their side on a glass slide under a dissecting microscope. The gonopodium was swung forwards and pressure was gently applied to the male's abdomen to expel sperm. Using a $10 \mu \mathrm{l}$ pipette we transferred the stripped ejaculate to a microcentrifuge tube containing a known volume $(100-300 \mu \mathrm{l})$ of saline solution $(0.9 \% \mathrm{NaCl})$.

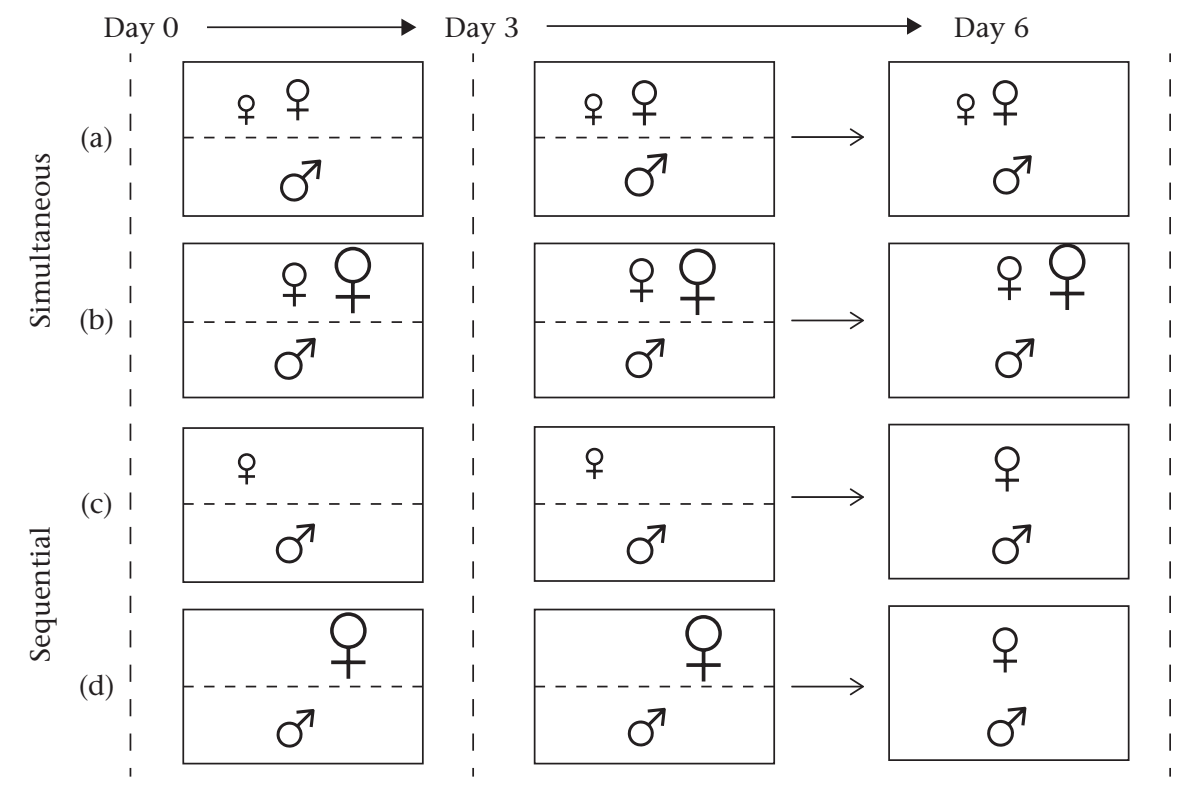

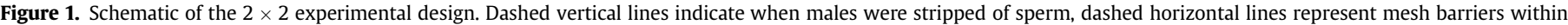

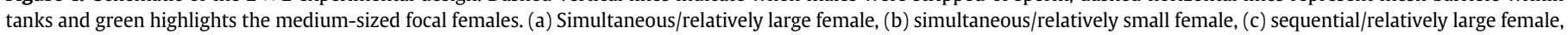
(d) sequential/relatively small female. 


\section{Collecting Sperm from Females}

We anaesthetized each female within 10 min of the behavioural trial and retrieved sperm from her gonoduct (see Pilastro \& Bisazza, 1999; Pilastro, Giacomello, \& Bisazza, 1997). A glass micropipette was used to flush the female's gonoduct with $30 \mu \mathrm{l}$ of saline solution $(0.9 \% \mathrm{NaCl})$.

\section{Sperm Number}

We counted sperm following the methods in Evans (2009). Briefly, sperm samples were vortexed for 1 min to break up sperm bundles and to evenly distribute sperm throughout the sample. Then $5 \mu$ l of the sample was placed on a Neubauer haemocytometer under x400 magnification (Kiyowa, Medilux-12 microscope). We photographed five cells of the haemocytometer so that sperm could later be counted blind to treatment. The five counts were averaged and the total number of sperm per male was then calculated by taking into account the concentration of the sample.

\section{Ethical Note}

This research was approved by the Australian National University Animal Ethics Committee (Approval no. A2011/64). We anaesthetized fish using an ice slurry prior to photographs and sperm collections because this method has been shown to be an ethical and effective method for anaesthetising small warm-water fish (Blessing, Marshall, \& Balcombe, 2010). Further, it allows quick and easy handling of fish during these procedures because no special protection is needed for the experimenter. This reduces potential stress for fish arising from these procedures. Sperm were collected from each male $(N=128)$ a total of three times over a 7day period during the experiment. Sperm were collected from females $(N=192)$ once during the experiment. All fish were monitored twice daily and stressed or dead fish were removed from the experiment $(N=6)$. The mortality rate of $<2 \%$ observed during the experiment was comparable to normal laboratory mortality levels. After the experiment males were returned to stock tanks whereas females were euthanized in an overdose of clove oil (Cunha \& Rosa, 2006).

\section{Data Analysis}

We used GLMs with appropriate error structures to investigate how mate encounter type and the focal female's relative size influenced (1) the number of copulation attempts with her, (2) whether a male inseminated the focal female or not and (3) how many sperm the male allocated to the focal female. All models included encounter type, the relative size of the focal female (categorical) and the absolute size of the focal female (continuous) as fixed effects. We also included the two-way interactions between encounter type, relative female size and absolute female size in the models. All GLMs were conducted using R v 3.1.0 (The R Foundation for Statistical Computing, Vienna, Austria, http://www.r-project. org). The total sample size was 122 for the number of attempted copulations and insemination success (sequential/relatively large female: $N=29$; sequential/relatively small female: $N=29$; simultaneous/relatively large female: $N=32$; simultaneous/relatively small female: $N=32$ ). Sample sizes were lower than the number of trios set up $(N=128 ; 32$ in each treatment $)$ due to missing data and mortality. Our analysis of the number of sperm transferred to a female was restricted to trials in which the focal female received sperm (sequential/relatively large female: $N=16$; sequential/relatively small female: $N=16$; simultaneous/relatively large female: $N=17$; simultaneous/relatively small female: $N=17$ ).

\section{RESULTS}

\section{Copulation Attempts}

The number of copulation attempts directed towards the focal female differed depending on her relative size and the type of encounter (interaction: $t_{1,109}=-3.847, P<0.001$ ). Males attempted to copulate significantly more often with a relatively larger than smaller focal female when females were presented simultaneously $\left(t_{1,56}=-3.532, P<0.001\right)$, but there was no effect of relative female size on the number of attempts when females were presented sequentially $\left(t_{1,56}=1.165, P=0.249\right.$; Fig. $\left.2 \mathrm{a}\right)$. The number of
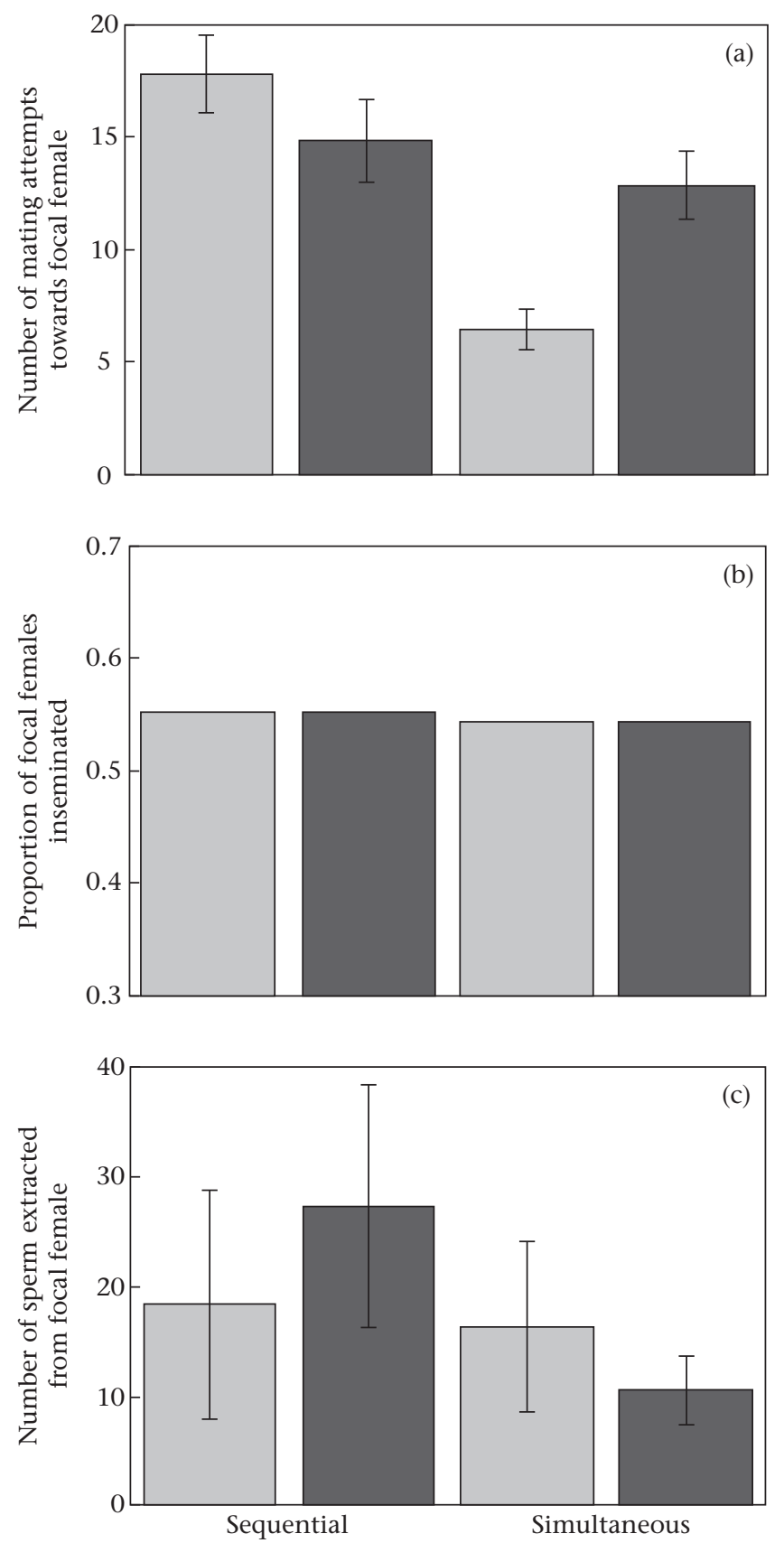

Figure 2. The effect of mate encounter type (sequential versus simultaneous) and the focal female's relative size on (a) the number of mating attempts a male directed towards the focal female (mean $\pm \mathrm{SE}$ ), (b) whether the focal female was inseminated or not and (c) the number of sperm allocated to the focal female (mean $\pm S E$ ). Light bars represent relatively small focal females and dark bars relatively large focal females. 
copulations directed towards the focal female also depended on her absolute size $\left(t_{1,109}=2.180, P=0.031\right)$ and this effect was not mediated by the type of encounter (interaction: $t_{1,109}=-0.339$, $P=0.736$ ) or the relative size of the focal female (interaction: $t_{1,109}=-0.877, P=0.382$ ). There was no main effect of female relative size or encounter type on the number of copulation attempts with the focal female (relative size: $t_{1,109}=1.013, P=0.313$; encounter type: $t_{1,109}=0.290, P=0.772$ ).

\section{Insemination Success and Sperm Transfer}

Neither the focal female's relative size nor her absolute size influenced the likelihood of insemination (relative size: $t_{1,109}=-1.467, P=0.145$; absolute size: $t_{1,109}=0.565, P=0.573$ ) or, for females that were inseminated, the mean number of sperm recovered from the reproductive tract (relative size: $t_{1,59}=-0.061$, $P=0.952$; absolute size: $t_{1,59}=-0.152, P=0.880$ ). Encounter type also had no effect on the likelihood that the focal female was inseminated $\left(t_{1,109}=1.019, P=0.310\right)$ or, if she was, the mean number of sperm recovered from her reproductive tract $\left(t_{1,59}=-0.714, P=0.478\right)$. There was no interaction between the focal females' relative size and encounter type for either insemination success $\left(t_{1,110}=-0.132, P=0.895\right)$ or the number of sperm recovered $\left(t_{1,59}=0.795, P=0.429\right.$; Fig. $\left.2 \mathrm{~b}, \mathrm{c}\right)$. There was no interaction between the focal females' absolute size and encounter type for either insemination success $\left(t_{1,109}=-1.002, P=0.318\right)$ or the number of sperm recovered $\left(t_{1,59}=0.591, P=0.557\right)$. There was also no interaction between the focal females' relative size and their absolute size for either insemination success $\left(t_{1,109}=1.508\right.$, $P=0.135)$ or the number of sperm recovered $\left(t_{1,59}=0.000\right.$, $P=1.000)$.

\section{DISCUSSION}

Males can afford to be choosier when their encounter rate with potential mates is high. This is because mate rejection then has a smaller effect on their actual mating rate (Jennions \& Kokko, 2014). The simultaneous presence of potential mates is indicative of a high mate encounter rate, so one might predict that males are less choosy when they encounter potential mates sequentially (and with a long interval between encounters). In addition, choice between simultaneously available mates carries no opportunity cost (Barry \& Kokko, 2010). If mate encounter rates vary then selection might favour male phenotypic plasticity in mating preferences based on cues that predict the prevailing social conditions (Svensson et al., 2010). Specifically, males should prefer highquality females when females are frequently encountered (e.g. simultaneously), but show no preferences when females are rarely encountered. Our results support this prediction. Male G. holbrooki attempted to mate with relatively large females more often than relatively small females when presented with two females simultaneously. This agrees with several earlier studies on G. holbrooki (e.g. Bisazza et al., 1989; Callander et al., 2012; Mautz \& Jennions, 2011; Wong \& McCarthy, 2009; but see McPeek, 1992). In contrast, males showed no such preference when presented with two females sequentially. Furthermore, our results show that males directed more copulations towards absolutely larger focal females (even within the restricted 'medium' size range used here) and that unlike the case for relative female size this pattern existed whether females were encountered sequentially or simultaneously. However, despite males directing more copulation attempts at relatively larger females in simultaneous encounter trials and absolutely larger females overall this increased neither insemination success nor the number of sperm transferred.
Our experiment monitoring male mating attempts supports the claim that males are less choosy when females are encountered sequentially rather than simultaneously (Fig. 2a), although this is not a general trend across taxa (meta-analysis: Dougherty \& Shuker, 2014). We cannot currently identify the proximate cause of this result. The encounter treatments should have generated a difference in perceived mate availability (and hence the perceived costs of rejecting the current mate), but they could also have affected a male's ability to discriminate between females. In a simultaneous encounter, males can directly compare the size of females, but cannot do so when females are encountered sequentially. Thus, both perceived mate availability and changes in discrimination ability could explain why a preference for relatively larger females was only seen in simultaneous encounters. Teasing apart these two effects is difficult, but possible. For example, Jordan and Brooks (2012) initially manipulated both how males encountered potential mates and the level of variation in female size in guppies, Poecilia reticulata. They then quantified male choice during sequential encounters. Males had stronger preferences for larger females if they had previously experienced greater variation in female size. Crucially, however, this effect was stronger for males that had previously encountered females simultaneously rather than sequentially (i.e. there was an interaction). This suggests that male choice is modified by perception of mate availability, but that discrimination between females based on size is reduced if mates are encountered sequentially (Jordan \& Brooks, 2012).

There are at least two other potential explanations for the difference in male mating preferences between encounter types that we observed. First, during sequential encounters males had not seen the focal female before, whereas they had in the simultaneous encounters. In many species males prefer unfamiliar females (e.g. Kelley, Graves, \& Magurran, 1999; LaDage \& Ferkin, 2006). This does not appear to be the case in mosquitofish, however, as males do not prefer novel females unless they have previously mated with them (Vega-Trejo et al., 2014). We suggest that a preference for unfamiliar females is unlikely to explain our results. Second, in the simultaneous encounters there was an opportunity for female-female interactions to affect male mating behaviour that was absent in the sequential encounters. Some studies suggest that male mate choice might be more strongly related to female dominance than female size or that larger females might restrict male access to small females (Chen, Beekman, \& Ward, 2011). Female-female interactions could explain why a mating preference for relatively larger females was only seen in the simultaneous encounters. We suggest that this is unlikely, however, as we did not observe any overtly aggressive interactions between females during mating trials.

We predicted that a male preference for relatively larger females would be weaker in sequential encounters, but the complete absence of a detectable preference is surprising. Previous research has shown that during sequential encounters females choose males based on their attractiveness relative to those that they have previously encountered and often 'trade up' (e.g. Kozak, Head, Lackey, \& Boughman, 2013; Rebar, Zuk, \& Bailey, 2011). Similar trends have been seen for male choice during sequential encounters (e.g. Wong, Jennions, \& Keogh, 2004), but far less is known about how recent experience and relative female attractiveness influence male mate choice (but see Barrett et al., 2014; Jordan \& Brooks, 2012; Svensson et al., 2010). In G. holbrooki previous studies demonstrating male choice for larger females have all been based on simultaneous choice trials (e.g. Callander et al., 2012; Mautz \& Jennions, 2011; Wong \& McCarthy, 2009). However, male choice for larger females has been shown in 'no-choice' trials in other poeciliid species (e.g. P. reticulata: Ojanguren \& Magurran, 2004). This is a timely reminder of the dangers of extrapolating from simultaneous choice 
experiments to choice in the field where mates are often encountered sequentially (Wagner, 1998).

It is intriguing that male attempted copulation rate increased with the absolute size of focal females, irrespective of whether encounters were sequential or simultaneous, but only for relatively larger females when encounters were simultaneous. One explanation for the increased attempted copulation rate towards absolutely larger females is that larger females may be easier to approach due to their decreased manoeuvrability (Pilastro et al., 1997). However, that there were more attempted copulations towards relatively larger females only during simultaneous encounters suggests that males have an additional 'active' preference for larger females, but that, as noted above, this preference is modified by either the costs of choice or the ease of detecting size differences between females.

There was no evidence for greater sperm allocation to relatively or absolutely larger females in either simultaneous or sequential encounter trials (Fig. 2). This suggests that G. holbrooki males do not strategically allocate sperm based on female size. In mosquitofish, males do not transfer all of their sperm reserves in a single mating (Evans \& Pilastro, 2011). Thus, the opportunity cost of mating with smaller, less fecund females might be low. During simultaneous encounters males were only presented with two females so they might have been able to allocate the optimal (hence equal) amount of sperm to both females because they did not exceed their sperm reserves. Furthermore, rival males were always absent, which should reduce the propensity for males to increase sperm allocation beyond the minimum necessary to ensure fertilization (metaanalysis: Kelly \& Jennions, 2011).

An alternative explanation invokes sexual conflict over sperm transfer. During simultaneous encounters with females males directed significantly more copulation attempts at larger females. Contrary to expectations, however, there was no resultant increase in either insemination success or the number of sperm transferred to larger females. Despite the high attempted copulation rate in our study (mean: 1.23 per min) only $54 \%$ of focal females were inseminated. This rate is similar to that reported in other studies (75\% insemination success after 30 min: Evans, Pierotti, \& Pilastro, 2003; 52\% insemination success after 24 h: Pilastro et al., 1997). This suggests that cryptic female mate choice partly determines whether and how many sperm are transferred during male copulation attempts. The lack of a relationship between attempted copulation rate and insemination success also highlights the need to be cautious of using proxies such as attempted copulation rate when inferring reproductive success.

\section{Conclusions}

Males directed significantly more copulation attempts towards relatively larger females when encountering females simultaneously rather than sequentially. This reflects a general, but nonsignificant, trend across all taxa (mean effect size $r=0.353$ versus 0.433: Dougherty \& Shuker, 2014). Previous studies in a few other species have, however, shown similar results, with males being significantly less choosy during no-choice trials than dichotomous choice trials (effect sizes in Dougherty \& Shuker, 2014). However, other species show high levels of choosiness even when mates are encountered sequentially. Lower consistency across species in how males respond to variation in encounter rate when compared to how females respond suggests that there may be sex differences in how mating ecology (e.g. natural encounter rate, density or sex ratio) influences the costs of rejecting mates. Further experiments investigating how variation in encounter rate influences male mate choice in carefully targeted species (e.g. to cover a range of natural mate encounter rates) would be useful to understand better why simultaneous versus sequential choice (i.e. experimentally 'two-choice' versus 'no-choice' tests) affects male mating preferences in some species but not in others.

\section{Acknowledgments}

We thank the ANU Animal Services team for fish maintenance. We also thank Susi Zajitschek for advice on extracting sperm from females, and Isobel Booksmythe for comments on the manuscript. This work was supported by the Australian Research Council (DP120100339).

\section{References}

Agrillo, C., Dadda, M., \& Serena, G. (2008). Choice of female groups by male mosquitofish (Gambusia holbrooki). Ethology, 114, 479-488.

Aspbury, A. S., \& Gabor, C. R. (2004). Differential sperm priming by male sailfin mollies (Poecilia latipinna): effects of female and male size. Ethology, 110, 193-202.

Barrett, L. T., Evans, J. P., \& Gasparini, C. (2014). The effects of perceived mating opportunities on patterns of reproductive investment by male guppies. PLoS One, 9, e93780.

Barry, K. L., \& Kokko, H. (2010). Male mate choice: why sequential choice can make its evolution difficult. Animal Behaviour, 80, 163-169.

Bisazza, A. (1993). Male competition, female mate choice and sexual dimorphism in poeciliid fishes. Marine Behaviour and Physiology, 23, 257-286.

Bisazza, A., Marconato, A., \& Marin, G. (1989). Male mate preferences in the mosquitofish Gambusia holbrooki. Ethology, 83, 335-343.

Bisazza, A., \& Marin, G. (1995). Sexual selection and sexual size dimporphism in the eastern mosquitofish Gambusia holbrooki (Pisces Poeciliidae). Ethology Ecology \& Evolution, 7, 169-183.

Blessing, J. J., Marshall, J. C., \& Balcombe, S. R. (2010). Humane killing of fishes for scientific research: a comparison of two methods. Journal of Fish Biology, 76, $2571-2577$.

Bonduriansky, R. (2001). The evolution of male mate choice in insects: a synthesis of ideas and evidence. Biological Reviews, 76, 305-339.

Booksmythe, I., Backwell, P. R. Y., \& Jennions, M. D. (2013). Competitor size, male mating success and mate choice in eastern mosquitofish, Gambusia holbrooki. Animal Behaviour, 85, 371-375.

Booksmythe, I., Jennions, M. D., \& Backwell, P. R. Y. (2011). Male fiddler crabs prefer conspecific females during simultaneous, but not sequential, mate choice. Animal Behaviour, 81, 775-778.

Callander, S., Backwell, P. R. Y., \& Jennions, M. D. (2012). Context-dependent male mate choice: the effects of competitor presence and competitor size. Behaviora Ecology, 23, 355-360.

Chen, T., Beekman, M., \& Ward, A. J. W. (2011). The role of female dominance hierarchies in the mating behaviour of mosquitofish. Biology Letters, 7, 343-345.

Constanz, G. D. (1989). Reproductive biologyof poeciliid fishes. In G. K. Meffe, \& F. F. Snelson, Jr. (Eds.), Ecology and evolution of livebearing fishes (Poeciliidae) (pp. 33-50). Englewood Cliffs, NJ: Prentice Hall.

Cornwallis, C. K., \& Birkhead, T. R. (2006). Social status and availability of females determine patterns of sperm allocation in the fowl. Evolution, 60, 1486-1493.

Cunha, F. E. A., \& Rosa, I. L. (2006). Anaesthetic effects of clove oil on seven species of tropical teleosts. Journal of Fish Biology, 69, 1504-1512.

Deaton, R. (2008). Factors influencing male mating behaviour in Gambusia affinus (Baird \& Girard) with a coercive mating system. Journal of Fish Biology, 72, 1607-1622.

Dougherty, L. R., \& Shuker, D. M. (2014). The effect of experimental design on the measurement of mate choice: a meta-analysis. Behavioral Ecology. http:// dx.doi.org/10.1093/beheco/aru125.

Edward, D. A., \& Chapman, T. (2011). The evolution and significance of male mate choice. Trends in Ecology \& Evolution, 26, 647-654.

Evans, J. P. (2009). No evidence for sperm priming responses under varying sperm competition risk or intensity in guppies. Naturwissenschaften, 96, 771-779.

Evans, J. P., Pierotti, M., \& Pilastro, A. (2003). Male mating behavior and ejaculate expenditure under sperm competition risk in the eastern mosquitofish. Behavioral Ecology, 14, 268-273.

Evans, J. P., \& Pilastro, A. (2011). Postcopulatory sexual selection. In J. P. Evans, A. Pilastro, \& I. Schlupp (Eds.), Ecology and evolution of poeciliid fishes (pp. 197-208). Chicago, IL: The University of Chicago Press.

Hoysak, D. J., \& Godin, J.-G. J. (2007). Repeatability of male mate choice in the mosquitofish, Gambusia holbrooki. Ethology, 113, 1007-1018.

Jennions, M. D., \& Kokko, H. (2014). Sexual selection: mate choice. In J. Losos, D. Baum, D. J. Futuyma, H. Hoekstra, R. Lenski, A. Moore, et al. (Eds.), The Princeton guide to evolution (pp. 647-654). Princeton, NJ: Princeton University Press.

Jordan, L. A., \& Brooks, R. C. (2012). Recent social history alters male courtship preferences. Evolution, 66, 280-287.

Kahn, A. T., Kokko, H., \& Jennions, M. D. (2013). Adaptive sex allocation in anticipation of changes in offspring mating opportunities. Nature Communications, 4 1603. 
Kekalainen, J., Huuskonen, H., Tuomaala, M., \& Kortet, R. (2010). Both male and female sexual ornaments reflect offspring performance in a fish. Evolution, 64, 3149-3157.

Kelley, J. L., Graves, J. A., \& Magurran, A. E. (1999). Familiarity breeds contempt in guppies. Nature, 401, 661-662.

Kelly, C. D., \& Jennions, M. D. (2011). Sexual selection and sperm quantity: metaanalyses of strategic ejaculation. Biological Reviews, 86, 863-884.

Kozak, G. M., Head, M. L., Lackey, A. C. R., \& Boughman, J. W. (2013). Sequential mate choice and sexual isolation in threespine stickleback species. Journal of Evolutionary Biology, 26, 130-140.

LaDage, L. D., \& Ferkin, M. H. (2006). Male Leopard geckos (Eublepharis macularis) can discriminate between two familiar females. Behaviour, 143, 1033-1049.

LeBas, N. R., Hockham, L. R., \& Ritchie, M. G. (2003). Nonlinear and correlationa sexual selection on 'honest' female ornamentation. Proceedings of the Royal Society B: Biological Sciences, 270, 2159-2165.

Lüpold, S., Manier, M. K., Ala-Honkola, O., Belote, J. M., \& Pitnick, S. (2010). Male Drosophila melanogaster adjust ejaculate size based on female mating status, fecundity, and age. Behavioral Ecology, 22, 184-191.

Matthews, I. M., Evans, J. P., \& Magurran, A. E. (1997). Male display rate reveals ejaculate characteristics in the Trinidadian guppy Poecilia reticulata. Proceedings of the Royal Society B: Biological Sciences, 264, 695-700.

Mautz, B. S., \& Jennions, M. D. (2011). The effect of competitor presence and relative competitive ability on male mate choice. Behavioral Ecology, 22, 769-775.

McPeek, M. A. (1992). Mechanisms of sexual selection operating on body size in the mosquitofish (Gambusia holbrooki). Behavioral Ecology, 3, 1-12.

Nordeide, J. T., Kekäläinen, J., Janhunen, M., \& Kortet, R. (2013). Female ornaments revisited-are they correlated with offspring quality? Journal of Animal Ecology, $82,26-38$.

O'Dea, R. E., Jennions, M. D., \& Head, M. L. (2014). Male body size and condition affects sperm number and production rates in mosquitofish, Gambusia holbrooki. Journal of Evolutionary Biology, 27, 2739-2744.

Ojanguren, A. F., \& Magurran, A. E. (2004). Uncoupling the links between male mating tactics and female attractiveness. Biology Letters, 271, s427-s429.

Pack, A. A., Herman, L. M., Spitz, S. S., Hakala, S., Deakos, M. H., \& Herman, E. Y. K. (2009). Male humpback whales in the Hawaiian breeding grounds preferentially associate with larger females. Animal Behaviour, 77, 653-662.

Parker, G. A. (1998). Sperm competition and evolution of ejaculates: towards theory base. In T. R. Birkhead, \& A. P. Moller (Eds.), Sperm competition and sexual selection (pp. 3-54). San Diego, CA: Academic Press.
Parker, G. A., \& Pizzari, T. (2010). Sperm competition and ejaculate economics. Biological Reviews, 85, 897-934.

Pilastro, A., \& Bisazza, A. (1999). Insemination efficiency of two alternative male mating tactics in the guppy (Poecilia reticulata). Proceedings of the Royal Society B: Biological Sciences, 266, 1887-1891.

Pilastro, A., Giacomello, E., \& Bisazza, A. (1997). Sexual selection for small size in male mosquitofish (Gambusia holbrooki). Proceedings of the Royal Society B: Biological Sciences, 264, 1125-1129.

Rebar, D., Zuk, M., \& Bailey, N. W. (2011). Mating experience in field crickets modifies pre and postcopulatory female choice in parallel. Behavioral Ecology, 22, 303-309.

Rowland, W. J. (1982). Mate choice by male sticklebacks, Gasterosteus aculeatus. Animal Behaviour, 30, 1093-1098.

Rubolini, D., Galeotti, P., Ferrari, G., Spairani, M., Bernini, F., \& Fasola, M. (2006). Sperm allocation in relation to male traits, female size, and copulation behaviour in a freshwater crayfish species. Behavioral Ecology and Sociobiology, 60, 212-219.

Svensson, P. A., Lehtonen, T. K., \& Wong, B. B. M. (2010). The interval between sexual encounters affects male courtship tactics in a desert-dwelling fish. Behavioral Ecology and Sociobiology, 64, 1967-1970.

Tigreros, N., Mowery, M. A., \& Lewis, S. M. (2014). Male mate choice favors more colorful females in the gift-giving cabbage butterfly. Behavioral Ecology and Sociobiology, 68, 1539-1547.

Vega-Trejo, R., O'Dea, R. E., Jennions, M. D., \& Head, M. L. (2014). The effects of familiarity and mating experience on mate choice in mosquitofish, Gambusia holbrooki. Behavioral Ecology, 25, 1205-1211.

Verrell, P. A. (1995). Males choose larger females as mates in the salamander Desmognathus santeetlah. Ethology, 99, 162-171.

Wagner, W. E. (1998). Measuring female mating preferences. Animal Behaviour, 55, 1029-1042

Wedell, N., Gage, M. J. G. \& Parker, G. A. (2002). Sperm competition, male prudence and sperm-limited females. Trends in Ecology \& Evolution, 17, 313-320.

Wilson, R. S. (2005). Temperature influences the coercive mating and swimming performance of male eastern mosquitofish. Animal Behaviour, 70, 1387-1394.

Wong B. B. M., Jennions, M. D., \& Keogh, J. S. (2004). Sequential male mate choice in a fish, the Pacific blue-eye Pseudomugil signifer. Behavioral Ecology and Sociobiology, 56, 253-256.

Wong, B. B. M., \& McCarthy, M. (2009). Prudent male mate choice under perceived sperm competition risk in the eastern mosquito fish. Behavioral Ecology, 20, $278-282$. 